\title{
La construction de l'Europe et la politique linguistique de la France
}

Le cas basque

Jean Haritschelhar

\section{OpenEdition}

\section{Journals}

Édition électronique

URL : http://journals.openedition.org/ries/4234

DOI : $10.4000 /$ ries.4234

ISSN : 2261-4265

\section{Éditeur}

Centre international d'études pédagogiques

Édition imprimée

Date de publication : 1 septembre 1994

Pagination : 85-92

ISSN : 1254-4590

\section{Référence électronique}

Jean Haritschelhar, "La construction de l'Europe et la politique linguistique de la France », Revue internationale d'éducation de Sèvres [En ligne], 03 | 1994, mis en ligne le 17 avril 2015, consulté le 01 mai 2019. URL : http://journals.openedition.org/ries/4234 ; DOI : 10.4000/ries.4234

Ce document a été généré automatiquement le 1 mai 2019.

(c) Tous droits réservés 


\section{La construction de l'Europe et la politique linguistique de la France}

Le cas basque

Jean Haritschelhar

1 C'est au cours des années 1980 que le Parlement européen, tout comme le Conseil de l'Europe, se sont préoccupés du sort des langues régionales ou minoritaires. Les résolutions - car l'un et l'autre ne peuvent que proposer des résolutions - se sont succédées depuis celle du Parlement européen du 16 octobre 1981 sur une charte communautaire des langues et cultures régionales jusqu'à la Charte européenne des langues régionales ou minoritaires, à laquelle le Conseil de l'Europe a conféré la forme juridique d'une Convention européenne soumise à signature des États à partir du 5 novembre 1992, et enfin la résolution Killilea, du nom du député irlandais qui l'a proposée et fait adopter le 9 février 1994 par le Parlement européen.

2 L'Espagne a signé la Charte européenne des langues régionales ou minoritaires. La France hésite, tergiverse et n'arrive pas à se décider. On comprend pourquoi il en est ainsi quand on examine le statut de la même langue des deux côtés de la Bidassoa, statut réel de langue officielle au sud, absence totale de statut au nord.

\section{L'Espagne et la liberté linguistique}

3 Le 20 novembre 1975, Franco meurt. Trois mois après, le 26 février 1976, au cours d'un conseil des ministres tenu à Barcelone (et non point à Madrid), sur proposition du ministre de l'éducation, l'Académie de la langue basque, Euskaltzaindia, est reconnue comme Académie royale. Le décret l'instituant paraît au Journal officiel du 26 février 1976. 
Ce geste hautement symbolique marque la fin de trente-huit années de répression contre la langue basque et amorce une nouvelle politique linguistique.

La Constitution espagnole confirme cette nouvelle politique. En effet, l'article 3 de la Constitution assure la reconnaissance des langues de la péninsule en trois paragraphes extrêmement clairs :

1. «Le castillan est la langue espagnole officielle de l'État. Tous les Espagnols ont le devoir de la connaître et le droit de l'utiliser.»

2. «Les autres langues espagnoles seront aussi officielles dans les diverses communautés autonomes en accord avec les statuts respectifs. »

3. «La richesse des diverses modalités linguistiques de l'Espagne est un patrimoine culturel qui fera l'objet de respect et protection spécifiques.»

5 Même si l'ensemble du Pays basque refuse la Constitution, la loi organique du 18 décembre 1979 relative au statut d'autonomie pour le Pays basque assure dans son article 16 l'officialisation de la langue basque: "L'euskara, langue propre du peuple basque aura, comme le castillan, le caractère de langue officielle en Euskadi et tous ses habitants ont le droit de connaître et utiliser les deux langues. » L'usage des deux langues est garanti, la non-discrimination assurée et l'Académie de la langue basque officialisée comme organisme consultatif en matière de langue dans les autres paragraphes du même article 6.

6 Le 24 novembre 1982, le parlement de la Communauté autonome basque vote la loi fondamentale de normalisation de l'euskara que le Président du gouvernement Carlos Garaikoetxea promulgue le jour même. Dans son titre préliminaire, l'article 2 déclare: «La langue propre au Pays basque est l'euskara. » L'article 3, conforme à la constitution espagnole ajoute: «Les langues officielles de la Communauté autonome du Pays basque sont le basque et le castillan. » Enfin, l'article 4 précise : « Les pouvoirs publics veilleront à adopter les mesures opportunes pour que personne ne subisse de discrimination pour un motif linguistique dans la Communauté autonome basque. »

7 Ainsi est affirmée la co-officialité du basque et du castillan sur le territoire de la Communauté autonome, ainsi est assurée la légalisation de l'euskara.

8 Le titre premier de cette loi fait référence aux droits des citoyens et aux devoirs de la puissance publique en matière linguistique : droit d'utiliser n'importe laquelle des deux langues, tant à l'écrit qu'à l'oral, devant l'administration, droit d'être enseigné dans les deux langues, droit de recevoir des publications périodiques, des programmes de radio et de télévision en langue basque, de développer des activités professionnelles, politiques ou syndicales en euskara, de s'exprimer en basque au cours de n'importe quelle réunion. La puissance publique se doit de garantir l'exercice de ces droits sur le territoire de la Communauté autonome.

Ces différents aspects sont repris et développés dans les titres suivants de la loi.

10 L'usage de la langue basque dans l'administration publique (titre II, articles 6 à 14) reconnu pour tous les citoyens, est adopté dans les transcriptions publiques, les registres, les imprimés et bulletins officiels de chacune des collectivités (gouvernement, "députations ", mairies, etc.). Devant la justice, le comparant pourra s'exprimer en basque et produire des écrits en basque. L'onomastique sera bilingue, aussi bien en ce qui concerne la toponymie que la signalisation routière, tandis que les imprimés et les avis dans les transports publics se feront dans les deux langues officielles. Pour répondre aux 
besoins du public, la « basquisation » du personnel administratif se fera progressivement en même temps que sera mis sur pied un corps de traducteurs et d'interprètes.

Dans l'enseignement (articles 15 à 21), le choix linguistique des élèves sera respecté et le gouvernement, ainsi que le parlement, s'engagent à généraliser le bilinguisme dans le système éducatif. Quelle que soit la langue d'enseignement choisie par les parents ou tuteurs, l'autre langue officielle sera obligatoire, de manière à parvenir à un bilinguisme, le gouvernement mettant en place des modèles adaptés à la situation sociolinguistique. Les écoles universitaires de formation des enseignants adapteront leurs programmes afin de parvenir au bilinguisme des futurs enseignants, tout en favorisant la «basquisation » du personnel en place.

qui concerne les moyens de communication de masse (articles 22 à 25), le droit des citoyens d'être informés en basque suppose un développement de cette langue, aussi bien à la télévision qu'à la radiodiffusion, dans la presse et autres publications, au cinéma, au théâtre, ainsi que dans tout mode de reproduction d'images et de sons.

Quant à l'utilisation sociale du basque (articles 26 à 29), la puissance publique prendra toutes les mesures nécessaires pour favoriser le développement de l'euskara dans les diverses activités commerciales, culturelles, associatives, sportives, religieuses, etc. Ainsi sera favorisée la publicité en basque et un programme d'alphabétisation et de « rebasquisation » des adultes sera mis sur pied.

14 Le cadre législatif devient une réalité. Il ne reste plus qu'à appliquer la loi. Pour cela sont créés deux organismes :

- Euskararen aholku batzordea, c'est-à-dire une commission consultative, une sorte de Haut conseil de la langue basque, créée le 11 janvier 1982, destinée à donner son avis sur tout texte intéressant la normalisation de l'euskara;

- le Secrétariat de la politique linguistique, directement rattaché à la présidence du gouvernement, organisme chargé d'impulser des enquêtes, de proposer des textes qui feront ensuite l'objet de décrets ou d'arrêtés, de promouvoir la politique linguistique du gouvernement.

Dans l'enseignement (décret 138 du 11 juillet 1983), le bilinguisme est assuré à travers trois modèles :

- modèle A : enseignement en espagnol, étude du basque ;

- modèle B : enseignement bilingue, $50 \%$ en basque et $50 \%$ en espagnol ;

- modèle D : enseignement en basque, étude de l'espagnol.

Ces trois modèles vont de la maternelle à l'université. Le même décret portait aussi sur la formation des maitres. On assiste à une "basquisation" régulière du système scolaire. Ainsi, en 1982, 25 \% des enfants suivaient les modèles B et D, et $75 \%$ se trouvaient dans le modèle A. Dix ans plus tard, en 1992, 56 \% sont en modèles B et D, et 44 \% en modèle A.

La loi du 20 mai 1982 crée la radio-télévision basque (Euskal irrati telebista). Deux chaînes existent, l'une en basque, l'autre en espagnol, chacune émettant douze heures par jour.

En ce qui concerne la presse, il existe en langue basque des magazines et des revues, soit d'information locale, soit culturels, ainsi que des hebdomadaires et un quotidien, Euskaldunon Egunkaria, fondé en 1991. Le nombre de livres basques publiés ne cesse de croître chaque année, atteignant en 1993 le chiffre de mille (cent à peine à la fin du franquisme), sous forme de livres scolaires ou de création littéraire (poésie, roman, 
théâtre, essai, etc.). Le ministère de la culture subventionne aussi le cinéma ainsi que le théâtre ou tout autre mode d'expression artistique.

La tâche la plus importante concernait l'administration qui a toujours travaillé en espagnol. Pour «basquiser » l'administration, le ministère de la culture a fondé, le 4 septembre 1981, l'Institut pour l'alphabétisation et la rebasquisation des adultes (HABE) qui dispense des cours dans l'ensemble du Pays basque, en concurrence avec l'organisme privé AEK qui avait vu le jour sous l'égide de l'Académie dans les dernières années du franquisme. De même, a été créée par décret du 23 septembre 1986 une école de traducteurs juridico-administratifs, rattachée à l'Institut basque d'administration publique, lui-même créé par décret du 11 juillet 1983. L'institut a pour but la formation et le perfectionnement des fonctionnaires, l'introduction, la diffusion et la normalisation de l'euskara dans l'administration de la Communauté autonome, ainsi que la fixation du langage administratif en collaboration avec l'Académie de la langue basque, Euskaltzaindia

En matière de droits linguistiques liés aux autonomies, l'Espagne a précédé la politique linguistique européenne ; elle est, à n'en pas douter, un véritable modèle.

\section{La France et l'unité linguistique}

21 Depuis bientôt cinquante ans, tous les partis politiques formant un groupe à l'Assemblée nationale ont déposé une ou plusieurs propositions de loi sur les langues régionales langues de France en réalité - mais aucune d'entre elles n'a été mise à l'ordre du jour de l'Assemblée. Le dispositif législatif sur les langues de France n'a jamais dépassé le stade des promesses, y compris celles du candidat à la présidence de la République François Mitterrand (proposition 54).

Deux textes législatifs existent - devenus tous deux obsolètes. Le premier est la loi $\mathrm{n}$ -51-46 du 11 janvier 1951, dite "loi Deixonne", du nom de son rapporteur, loi sur l'enseignement des langues et dialectes locaux. Cette loi qui prenait en compte le basque, le breton, le catalan et l'occitan autorise l'organisation de cours facultatifs d'une heure par semaine dans le premier et le second degré. La notion de facultatif est comprise tant pour les élèves (ou leurs parents) que pour les maîtres.

Le deuxième texte est la loi n 75-620 du 11 juillet 1975, dite "loi Haby », du nom du ministre de l'éducation nationale qui l'a fait voter. L'article 12 stipule «qu'un enseignement facultatif des langues et cultures régionales peut être organisé tout au long de la scolarité ».

La circulaire $n^{\circ} 75-426$ du 21 novembre 1975 permet l'organisation de stages de langues et cultures régionales et la circulaire $\mathrm{n}^{\circ} 76-123$ du 29 mars 1976 prend en compte dans l'enseignement les patrimoines linguistiques et culturels français correspondant aux langues régionales, les mêmes qu'en 1951 auxquelles s'est ajouté le corse. Ces langues (une heure par semaine) peuvent être enseignées à l'école maternelle - enseignement préélémentaire - ainsi que dans le premier et le second degré. En outre, la création de postes de conseillers pédagogiques est prévue.

Il faut attendre la circulaire ministérielle n ${ }^{\circ} 82-261$ du 21 juin 1982, dite circulaire Savary, ministre de l'éducation nationale du gouvernement socialiste, pour voir affirmé l'engagement de l'État en ce qui concerne l'enseignement des cultures et langues régionales dans le service public de l'éducation nationale, enseignement qui bénéficiera 
d'un véritable statut et qui sera basé sur le volontariat des élèves et des enseignants dans le respect de la cohérence du service public.

Cet enseignement sera dispensé de la maternelle à l'université, non pas comme une matière marginale mais comme une matière spécifique. Outre l'enseignement spécifique de la langue, modulable de une à trois heures par semaine, certaines activités d'éveil à la culture régionale pourront être conduites dans la langue régionale. La voie est ouverte vers des classes bilingues évoquées dans la circulaire: "Enfin seront étudiées les conditions dans lesquelles pourraient être créées des classes expérimentales bilingues tenant compte des expériences déjà engagées dans certaines régions et faisant appel aux compétences qu'elles ont ainsi révélées. »

Tels sont les textes en vigueur en France qui, depuis quarante ans, régissent l'enseignement des langues de l'Hexagone autres que le français. En outre, les langues de France font l'objet d'une option de trois heures hebdomadaires en classes de quatrième et de troisième, comme toute autre langue vivante II. L'épreuve orale facultative du baccalauréat est étendue aux baccalauréats de technicien, ainsi qu'à certains brevets de technicien supérieur.

28 Cette avancée indéniable a permis, pour la langue basque, de proposer dans l'enseignement public, ainsi que dans l'enseignement privé confessionnel, deux filières dans le cycle élémentaire :

- la filière A: depuis 1969, un corps d'itinérants dispense trois heures d'enseignement hebdomadaire dans les diverses classes d'une école ;

- la filière B : il s'agit, conformément à la circulaire Savary, d'un enseignement bilingue 14 heures en français, 12 heures en basque, les matières enseignées en basque étant les mathématiques, les sciences naturelles, l'histoire, une partie de l'éducation musicale, artistique et sportive, les enseignants étant différents selon la langue; la première classe s'est ouverte en 1983-1984.

En outre, la filière $\mathrm{D}$ (celle des ikastolas, écoles basques) est impulsée par l'association Seaska (le berceau), ainsi nommée parce qu'elle a instauré cette filière à partir de la maternelle, en 1969-1970. La langue d'enseignement est le basque, avec étude du français à partir du cours élémentaire $1^{\text {re }}$ année ( 3 heures hebdomadaires, étendues à 9 heures, c'est-à-dire un tiers du temps aux cours moyens $1^{\mathrm{re}}$ et $2^{\mathrm{e}}$ année). Toutes les matières sont enseignées en basque dans le cycle élémentaire. Cette filière se poursuit dans un collège et un lycée à charge de l'association.

De 1969 à 1982, aucune subvention d'État n'a été accordée à l'association. À partir de 1982 et progressivement, des subventions d'abord, des prises en charge d'enseignants ensuite, seront octroyées par le biais de conventions. Actuellement, des négociations sont en cours avec le ministère de l'éducation nationale. Ces écoles pourraient obtenir le même statut que les écoles privées et entrer dans le cadre de la loi Debré. En conséquence, elles seraient dotées du statut d'écoles privées non confessionnelles.

31 Alors que les autres langues de France obtenaient les diplômes officiels de l'enseignement supérieur, permettant en particulier la formation des maîtres, ce n'est qu'en 1989 que fut accordé le DEUG de basque, en 1991 la licence et la maîtrise et, en 1993, le CAPES, ces enseignements étant dispensés dans le département interuniversitaire d'études basques de Bayonne, créé conjointement par les universités de Bordeaux III et de Pau.

32 Il faut reconnaître, en ce qui concerne l'enseignement, que l'État français remplit les conditions requises par la Charte européenne des langues régionales ou minoritaires du 
Conseil de l'Europe, soumise à la signature à partir du 5 novembre 1992. Mais l'État français ne remplit aucune des conditions exigées par l'article 7 consacré aux services publics, autorités administratives et justice. Aucun texte officiel n'est bilingue, l'administration et la justice ne connaissent que le français et ce d'autant plus que l'article 2 de la Constitution déclare, depuis la dernière révision, que «la langue de la République est le français ».

Quant à l'article 8 sur les médias, il suffit de savoir quels sont les temps d'antenne pour juger à quel point l'État se désintéresse de la question. Le seul organe de presse basque, l'hebdomadaire Herria, ne bénéficie d'aucune subvention.

Radio France Pays Basque émet en basque une heure par jour pendant cinq jours, ce qui fait environ 250 heures dans l'année, ce que Euskadi irratia réalise en quinze jours à SaintSébastien. Il faut reconnaître cependant que les trois radios privées émettant en basque reçoivent quelques subsides de l'État. À la télévision, dans le décrochage de six minutes de France 3-Euskal Herri, l'expression en basque dure un maximum de une minute et demie et, même en comptant cinq jours par semaine, ce qui n'est pas toujours le cas, on arriverait à un total de six heures dans l'année, c'est-à-dire l'équivalent d'une demi-journée en Espagne sur la chaîne Euskal Telebista, qui émet douze heures par jour. Quand on songe que le paragraphe d) de cet article déclare «ne poser aucune entrave à la réception des programmes médiatiques des pays voisins de même langue et culture et, si possible, favoriser une telle réception » et que le gouvernement ou, à défaut, le CSA met toutes les entraves possibles à la réception d'Euskal Telebista, on peut juger du non-vouloir et de l'esprit d'intolérance qui existent dans les pouvoirs publics.

\section{Où est l'erreur, où est la vérité ?}

Même si la langue basque est minoritaire en Pays basque sud, le dispositif législatif en vigueur, qui lui donne le statut de langue officielle, fait que la politique linguistique de l'Europe, à travers les résolutions ou la Charte, n'apporte rien à la communauté autonome d'Euskadi, alors qu'elle peut avoir quelque effet pour la Navarre, surtout dans sa zone non-bascophone. La législation, si elle est une condition nécessaire, n'est cependant pas une condition suffisante. En effet, l'application de la loi dépend des décrets et plus encore, de la population qui la respecte. Tel est le défi que se lancent le gouvernement et le peuple basque. Lire et étudier, parler et écouter, travailler et créer, en un mot, vivre en basque, tel est l'objectif clair dont on peut mesurer les progrès au cours des dix dernières années. La conscience linguistique s'aiguise chaque jour davantage et les nouvelles générations enseignées dans le nouveau système éducatif arrivent à l'âge adulte et accentueront le désir de récupération de la langue basque dont on sait qu'elle fut particulièrement méprisée et pourchassée pendant l'ère franquiste.

Par contre, la résolution Killilea du 9 février 1994 interpelle le gouvernement français : elle souligne la nécessité pour les États, donc pour la France, de reconnaître leurs minorités linguistiques, d'accorder un statut légal approprié aux diverses langues et donc à la langue basque; elle souhaite l'utilisation de ces langues "au niveau de l'enseignement, de la justice, de l'administration publique, des médias, de la toponymie et des autres secteurs de la vie publique et culturelle » et invite les gouvernements des États membres à signer la Convention et leurs parlements à la ratifier d'urgence, en choisissant à tout moment d'appliquer les paragraphes qui répondent le mieux aux besoins et aux aspirations des communautés linguistiques concernées. 
cest là que le bât blesse car trente-cinq paragraphes doivent être choisis parmi les dispositions de la partie III, dont au moins douze parmi un certain nombre de paragraphes énoncés. Les paragraphes en question portent sur l'enseignement, les services publics, les autorités administratives et la justice, les médias, etc. Imagine-t-on la possibilité de formuler à l'administration une demande rédigée en langue basque? Que les services judiciaires garantissent la possibilité pour l'accusé de s'exprimer en euskara? De garantir l'existence d'une chaîne de télévision diffusant principalement ou totalement en basque ? Ou, pour le moins, assurant la diffusion régulière et dans tous les domaines d'émissions télévisées en langue basque? Ou enfin, pour en terminer avec cette série, de reconnaître la validité des actes juridiques formulés en basque?

Il faudra une importante évolution des mentalités pour que le gouvernement français accepte de signer la Charte européenne des langues régionales et minoritaires. Et pourtant, c'est un même peuple, c'est une même langue qui se situe des deux côtés d'une frontière. De quel côté des Pyrénées est la vérité, de quel côté l'erreur? Il est évident que la patrie des droits de l'Homme n'est pas la patrie des droits linguistiques.

\section{RÉSUMÉS}

L'analyse comparative du statut de la langue basque et de la législation relative à son usage dans la vie publique de part et d'autre des Pyrénées met en évidence les approches divergentes de la politique linguistique en France et en Espagne.

\section{INDEX}

Index géographique : France, Espagne, Pays basque, Europe

Mots-clés : politique linguistique, langue régionale, langue basque

\section{AUTEUR}

\section{JEAN HARITSCHELHAR}

Professeur émérite de l'université Michel de Montaigne-Bordeaux III, président d'Euskaltzaindia / Académie de la langue basque. 